\title{
BALANCE ENTRE RIESGOS Y BENEFICIOS DEL TAMIZAJE MAMOGRÁFICO DE CÁNCER DE MAMA ¿APOYARÍA SU RECOMENDACIÓN EN MUJERES PERUANAS?
}

\author{
Margarita Posso 1,2,a, Teresa Puig',3,b, Xavier Bonfill ${ }^{1,2,3, c}$
}

\begin{abstract}
RESUMEN
El objetivo de este estudio es valorar, en base a un análisis crítico y estructurado de la evidencia, si el tamizaje mamográfico de cáncer de mama en Perú es una intervención recomendable. El análisis se realizó utilizando los criterios propuestos por el sistema GRADE (Grading of Recommendations Assessment, Development and Evaluation). Se realizó una búsqueda de la literatura en Medline y en otras fuentes de información nacional (literatura gris). Para el desarrollo de la recomendación, a favor o en contra del tamizaje, se utilizó la mejor evidencia disponible sobre los beneficios, riesgos, costos y preferencias de la población. Los resultados incluyeron cinco revisiones sistemáticas (RS) que evaluaron los riesgos y beneficios del tamizaje; dos evaluaciones económicas realizadas en Perú y un estudio que valoró indirectamente las preferencias de las mujeres peruanas. La calidad de la evidencia a partir de las RS fue moderada a favor del tamizaje en mujeres de 50 a 69 años. El balance entre riesgos y beneficios mostró una mayor probabilidad de sobrediagnóstico comparado con el beneficio en la reducción de la mortalidad. La estrategia más costoefectiva fue la mamografía trienal. La percepción de barreras podría comprometer seriamente la participación de las mujeres. En conclusión, la recomendación a favor del tamizaje mamográfico en Perú es débil, más aun si se tienen en cuenta otras necesidades poblacionales más urgentes. En caso de llevarse a cabo, la estrategia de tamizaje más adecuada para la población peruana consistiría en realizar una mamografía trienal en mujeres de 50 a 69 años.
\end{abstract}

Palabras clave: Cribado; Neoplasias de la mama; Mamografía (fuente: DeCS BIREME).

\section{BALANCING RISKS AND BENEFITS OF MAMMOGRAPHY SCREENING FOR BREAST CANCER: WOULD YOU SUPPORT ITS RECOMMENDATION IN PERUVIAN WOMEN?}

\begin{abstract}
The aim of this study is to assess the balance between benefits and risks of population-based mammography screening programs in Peruvian women. We followed the criteria proposed by the GRADE (Grading of Recommendations Assessment, Development and Evaluation) working group. A generic search strategy for published studies was performed using Medline and other sources of national data (gray literature). The evidence of benefits, risks, costs and preferences of the population was used in order to state a recommendation in favour, or against, screening. We found five systematic reviews (SR) that evaluated the balance between benefit and risks, two economic evaluations based on Peruvian data, and one study that reported the preferences of Peruvian women. The quality of evidence of the SR was moderate in favour of screening in women aged 50-69 years. The balance of risks and benefits showed a higher probability of overdiagnosis compared with the reduction in mortality. The most cost-effective strategy was the triennial mammography. Perceived barriers could seriously compromise the participation of women. In conclusion, the recommendation of mammography screening for Peruvian women is weak, even more if we take into account other health necessities of the population. However, if implemented, triennial mammography in women aged 50-69 years could be the more suitable screening strategy
\end{abstract}

Key words: Straining; Breast Neoplasms; Mammography (source: MeSH NLM).

\footnotetext{
Universidad Autónoma de Barcelona, Barcelona, España.

Centro Cochrane Iberoamericano. Institut de Recerca Biomèdica Sant Pau (IIB Sant Pau), Barcelona, España.

Hospital de la Santa Creu i Sant Pau. Institut de Recerca Biomèdica Sant Pau (IIB Sant Pau), Barcelona, España.

Médica especialista en Medicina Preventiva y Salud Pública, MPH; ${ }^{\text {b }}$ Médica especialista en Medicina Preventiva y Salud Pública, MPH, PhD; ${ }^{\mathrm{c}}$ médico especialista en Oncología Médica, MPH, PhD

El presente artículo forma parte de la tesis doctoral titulada: "Cribado poblacional del cáncer de mama: aspectos relacionados con la efectividad y costeefectividad”, de la autoría de Margarita Posso. Departamento de Pediatría, Obstetricia y Ginecología y Medicina Preventiva. Universitat Autònoma de Barcelona. Barcelona, España.

Recibido: 07-08-14 Aprobado: 28-01-15
}

Citar como: Posso M, Puig T, Bonfill X. Balance entre riesgos y beneficios del tamizaje mamográfico de cáncer de mama ¿apoyaría su recomendación en mujeres peruanas?. Rev Peru Med Exp Salud Publica. 2015;32(1):117-28. 


\section{INTRODUCCIÓN}

A nivel mundial, con una tasa ajustada por edad de 43,3/100 000 habitantes, según cifras publicadas en 2012 por la Agencia Internacional de Investigación en Cáncer, el cáncer de mama ocupa el segundo lugar en frecuencia en mujeres (1). En Perú, según el Sistema Nacional de Vigilancia Epidemiológica, durante el periodo 2006-2011, el 16,6\% (11 271) del total de casos de cáncer diagnosticados en mujeres correspondió a pacientes con cáncer de mama (2), afectando principalmente a las mayores de 50 años $(80 / 100000){ }^{(1)}$. Así, en el país esta neoplasia es la tercera causa de mortalidad por cáncer, contando 10 defunciones por 100000 habitantes (1) y la supervivencia es de $70 \%$ a los 5 años ${ }^{(3)}$.

En Perú, aproximadamente un $40 \%$ de las pacientes son diagnosticadas en estadios avanzados lo que reduce las posibilidades terapéuticas y conlleva un peor pronóstico ${ }^{(3)}$. La edad promedio al diagnóstico es 54 años y a la muerte 58,4 años ${ }^{(3)}$. Esta rápida progresión de la enfermedad podría estar condicionada por el escaso acceso al tratamiento y su baja efectividad en los estadios avanzados pues solo un $69,5 \%$ de la población cuenta con un sistema de cobertura en salud ${ }^{(4)}$. Se ha estimado que en la población femenina el 1,2\% de los años de vida saludables perdidos debido a muerte prematura o discapacidad (AVISA) son atribuibles al cáncer de mama ${ }^{(3)}$. En general, las enfermedades no transmisibles representarían el $66 \%$ de los AVISA y en particular los tumores malignos serían responsables del $7 \%$ de la carga de enfermedad ${ }^{(5)}$.

Tal como lo establece la Organización Mundial de la Salud (OMS), las mujeres sintomáticas no se beneficiarían de un programa de tamizaje, sino más bien de un circuito de diagnóstico y tratamiento rápido ${ }^{(6)}$. Para la población asintomática la mamografía es el único método de tamizaje que se ha revelado eficaz. Métodos de tamizaje como la autoexploración mamaria o la exploración clínica no han demostrado grandes beneficios ${ }^{(5)}$. Los estudios disponibles muestran deficiencias en estas técnicas debido a su baja sensibilidad, alto porcentaje de falsos positivos, discontinuidad en la realización y variabilidad en la técnica ${ }^{(7-9)}$. Según un estudio realizado en Chile, durante el 2004-2007 se alcanzó un $65 \%$ de participación en el tamizaje clínico. A pesar de esta gran adherencia no se evidenció un incremento en el porcentaje de diagnósticos tempranos ni se redujo el porcentaje de estadios avanzados ${ }^{(10)}$.

Existen estudios cuyos resultados indican un efecto beneficioso del tamizaje mamográfico sobre la reducción en la mortalidad por cáncer de mama ${ }^{(11,12)}$ por lo que en muchos países se han implementado programas poblacionales ${ }^{(13,14)}$. Sin embargo, desde el momento en que se establecieron estos programas ha existido un intenso debate en relación a sus posibles beneficios y perjuicios. Clásicamente se han descrito consecuencias negativas en las mujeres con resultados falsos positivos ${ }^{(15,16)}$ y más recientemente el debate gira en torno al sobrediagnóstico. El sobrediagnóstico se ha definido como la detección de lesiones, incluidos algunos carcinomas, que debido a su historia natural nunca se habrían manifestado clínicamente (17). Los perjuicios derivados del sobrediagnóstico son frecuentes y conllevan un deterioro importante sobre la calidad de vida de las mujeres sometidas a otras pruebas diagnósticas invasivas $\mathrm{o}$ tratamientos quirúrgicos innecesarios ${ }^{(18,19)}$.

El gran número de publicaciones científicas en este tema ha generado una mayor necesidad de sistematizar la revisión de la literatura para extraer conclusiones válidas. Si bien las RS proporcionan información esencial sobre la eficacia del tamizaje mamográfico de cáncer de mama, el juicio para recomendar una intervención a nivel poblacional requiere un enfoque más amplio. En este sentido, la utilización crítica de la evidencia para orientar la toma de decisiones es necesaria, más aún si tenemos en cuenta que los recursos son siempre limitados.

Durante los últimos años el sistema GRADE viene siendo ampliamente utilizado para el desarrollo de recomendaciones (20). Las organizaciones que están adheridas al grupo de trabajo o que utilizan GRADE son referentes a nivel internacional como son la OMS, la Colaboración Cochrane, diferentes sociedades científicas norteamericanas y europeas, entre otros. GRADE propone clasificar la calidad de la evidencia y desarrollar recomendaciones de forma explícita, rigurosa y consistente. Bajo este enfoque las recomendaciones a favor o en contra de una intervención deben desarrollarse con base en la mejor evidencia disponible y siguiendo principalmente los siguientes criterios: 1) los beneficios o la eficacia, 2) los riesgos o seguridad, 3) los costos o utilización de recursos, y 4) las preferencias de la población (21).

En Perú, mediante el denominado "Plan Esperanza" el Estado ha priorizado el manejo del cáncer (22). La Coalición Multisectorial liderada por el Ministerio de Salud ha planteado como objetivo para el año 2016 realizar como mínimo una mamografía al $30 \%$ de las mujeres mayores de 40 años ${ }^{(23)}$. Con el objetivo de contribuir de manera cuantitativa a esta iniciativa, en este trabajo se han utilizado los criterios propuestos por el sistema GRADE para elaborar recomendaciones útiles para la toma de decisiones y adaptadas al contexto del país. 


\section{MÉTODO}

\section{ESTRATEGIA DE BÚSQUEDA}

Para identificar estudios sobre la eficacia y seguridad de la intervención se realizó una búsqueda de la literatura en Medline. Se combinaron los términos relacionados con cáncer de mama, programas poblacionales de tamizaje y eficacia (Tabla 1). Las evaluaciones económicas y los estudios de preferencias también se buscaron en Medline incorporando en la estrategia anterior los términos relacionados con Perú, costos y valoraciones. La búsqueda manual de literatura gris se realizó principalmente para identificar estudios de utilización de recursos y preferencias de la población. Se revisaron las páginas web del Ministerio de Salud, la Dirección General de Epidemiología, el Instituto Nacional de Salud, el Instituto Nacional de Enfermedades Neoplásicas y en el repositorio nacional de la Red Peruana de Tesis Digitales.

\section{ELECCIÓN DE LOS DESENLACES O RESULTADOS CRÍTICOS. EFICACIA Y SEGURIDAD}

Eficacia: el propósito del tamizaje es detectar precozmente las neoplasias y evitar que alcancen estadios más avanzados. Este adelantamiento en el momento del diagnóstico permitiría una intervención temprana y una disminución de la mortalidad por esta causa. Es por esto que la mejor forma de evaluar el impacto positivo del tamizaje mamográfico es midiendo la disminución en la mortalidad (17).

Seguridad: el sobrediagnóstico es el principal efecto indeseable en las mujeres participantes de un programa de tamizaje poblacional. Un cáncer sobrediagnosticado es aquel que de no ser por el tamizaje no hubiera alterado la vida de la mujer ${ }^{(17)}$. El impacto negativo de las pruebas adicionales y los tratamientos innecesarios en las mujeres sobrediagnosticadas supera el impacto negativo de otros efectos indeseables como son los resultados falsos negativos o falsos positivos, la radiación en el tórax, etc. ${ }^{(17)}$.

\section{EVALUACIÓN DE LA CALIDAD DE LA EVIDENCIA}

GRADE propone evaluar la calidad de la evidencia en su conjunto, independientemente de la calidad individual de cada uno de los estudios sobre la eficacia y seguridad. Así, para el desarrollo de este apartado, se valoraron de forma explícita y estructurada el riesgo de sesgo (limitaciones en los estudios), la inconsistencia, la dirección de la evidencia y la imprecisión de los desenlaces de interés. Se utilizaron las tablas GRADE para el resumen de la evidencia ${ }^{(21)}$.

\section{ANÁLISIS DEL USO DE RECURSOS}

Se respondió a la pregunta sobre si el uso de recursos compensa el beneficio neto esperado. Por tanto, para el desarrollo de este apartado se han incluido solo evaluaciones económicas completas en las cuales se valore de forma simultánea el uso de los recursos respecto al efecto incremental ganado con una intervención frente a otra. Las evaluaciones económicas varían respecto al contexto en el que se desarrollan por lo que en este estudio solo se han incluido evaluaciones económicas desarrolladas en Perú.

\section{ANÁLISIS DE LAS PREFERENCIAS DE LA POBLACIÓN}

GRADE propone evaluar si se puede asumir que las preferencias son similares en la población que va a recibir la intervención. En caso de ser homogénea a favor la recomendación sería fuertemente aconsejable.

Tabla 1. Estrategia de búsqueda en Ovid (http://gateway.ovid.com/).

1. Breast Neoplasms/

2. (Breast adj2 (neoplasm\$ or carcinoma $\$$ or tumour $\$$ or tumor $\$$ or cancer\$)).ti,ab.

3. 1 or 2

4. Mass Screening/

5. Mammographyl

6. (breast adj2 (screen\$ or program $\$$ or service $\$)$ ).ti,ab.

7. (mammogra $\$$ or mamogra $\$$ ).ti, ab.

8. (Breast adj2 (radiograph\$ or imaging or visualize or visualise or exam\$ or test\$)).ti,ab.

9. 4 or 5 or 6 or 7 or 8

10. (over-diagnosis OR over diagnosis OR overdiagnosis OR underdiagnosis OR underdiagnosis OR under diagnosis OR misdiagnosis OR mis-diagnosis OR over-treat\$ OR over treat\$ OR overtreat\$ OR under-treat\$ OR under treat\$ OR false positive OR false-positive OR harm\$ OR wound OR injur\$). ti,ab.

11. (life year gained OR QALY OR quality adjusted life year OR quality of life OR quality OR better life OR healthy life OR happy life OR mortal\$ OR death\$ OR dead\$ OR deceased\$ OR defunct\$ OR fallen OR departed OR breast cancer mortality OR breast-cancer mortality OR cancer mortality OR preventable death\$ OR preventable defunct\$). ti,ab.

12. 10 or 11

13. 3 and 9 and 12 
Tabla 2. Características de los ensayos clínicos aleatorizados (ECA) incluidos en las principales revisiones sistemáticas (RS) que evalúan los riesgos y beneficios del cribado mamográfico de cáncer de mama.

\begin{tabular}{|c|c|c|c|c|c|c|c|c|}
\hline \multirow[b]{2}{*}{$\begin{array}{c}\text { Revisión } \\
\text { sistemática }\end{array}$} & \multirow[b]{2}{*}{$\begin{array}{l}\text { Modelo de } \\
\text { metanálisis }\end{array}$} & \multicolumn{7}{|c|}{ Características de los estudios incluidos en el metanálisis } \\
\hline & & $\begin{array}{c}\text { ECA } \\
\text { (año de inicio) }^{\text {ref }}\end{array}$ & $\begin{array}{c}\text { Población } \\
\text { (n) }\end{array}$ & $\begin{array}{c}\text { Edad } \\
\text { (años) }\end{array}$ & Intervención & Control & $\begin{array}{c}\text { Intervalo }^{i} \\
\text { (meses) }\end{array}$ & $\begin{array}{c}\text { Duración }^{d} \\
\text { (años) }\end{array}$ \\
\hline \multirow{9}{*}{$\begin{array}{l}\text { Cochrane } \\
\text { Reviews }^{24}\end{array}$} & & Canadá I $(1980)^{28}$ & 50430 & $40-49$ & $M+E C+A E$ & $\mathrm{AE}$ & 12 & 5 \\
\hline & & Canadá II $(1980)^{29}$ & 39405 & $50-59$ & $M+E C+A E$ & $E C+A E$ & 12 & 5 \\
\hline & & Göteborg $(1982)^{30}$ & 52222 & $39-59$ & M & NC & 18 & 7 \\
\hline & Efecto fijo & $\begin{array}{l}\text { Kopparberg } \\
(1977)^{31}\end{array}$ & 57897 & $38-75$ & $M+A E$ & NC & $24-33$ & 7 \\
\hline & & Malmö $(1976)^{32}$ & 42238 & $45-69$ & M & NC & $18-24$ & 12 \\
\hline & & New York $(1963)^{33}$ & 62000 & $40-64$ & $M+E C$ & $\mathrm{NC}$ & 12 & 3 \\
\hline & & $\begin{array}{l}\text { Ostergötland } \\
(1978)^{34}\end{array}$ & 76970 & $38-75$ & $M+A E$ & $\mathrm{NC}$ & $24-33$ & 7 \\
\hline & & Stockholm $(1981)^{35}$ & 60800 & $39-65$ & M & $\mathrm{NC}$ & $24-28$ & 4 \\
\hline & & $\begin{array}{l}\text { UK age trial } \\
(1991)^{36}\end{array}$ & 160921 & $39-41$ & M & $\mathrm{NC}$ & 12 & 8 \\
\hline $\begin{array}{l}\text { UK National } \\
\text { Health Service }^{25}\end{array}$ & Efecto aleatorio & Igual al anterior* & & & & & & \\
\hline $\begin{array}{l}\text { Canadian } \\
\text { TFPHC }^{26}\end{array}$ & Efecto aleatorio & Igual al anterior* & & & & & & \\
\hline US PSTF $2009^{27}$ & Efecto aleatorio & Igual al anterior* & & & & & & \\
\hline
\end{tabular}

$\mathrm{ECA}=$ ensayo clínico aleatorizado, $\mathrm{M}=$ mamografía, $\mathrm{EC}=$ examen clínico, $\mathrm{AE}=$ autoexploración, $\mathrm{NC}=$ no cribado. ${ }^{*}$ Todas las $\mathrm{RS}$ incluyeron en el metanálisis los mismos ECA. IIntervalo de tiempo entre una prueba de cribado y la siguiente. 'Duración del estudio.

Para el desarrollo de este apartado se incorporaron referencias nacionales en las que se valoren de la forma más directa posible las preferencias de la población afectada.

\section{DESARROLLO DE LA RECOMENDACIÓN FINAL}

Considerando el balance entre riesgos y beneficios del uso de los recursos y las preferencias de la población, se desarrolló una recomendación a favor o en contra. La fuerza de la recomendación se basó en la incertidumbre de los resultados que afectan el sentido de la recomendación. Todo el proceso se estructuró utilizando la tabla propuesta por GRADE para la generación de recomendaciones ${ }^{(21)}$.

\section{RESULTADOS}

Se identificaron cinco RS, elaboradas por reconocidos referentes científicos como son la RS Cochrane (24), la del Servicio Nacional de Salud del Reino Unido ${ }^{(25)}$, las guías de práctica clínica de la Task Force canadiense (26) y estadounidense ${ }^{(27)}$ y la RS de la Red Europea de Programas de Tamizaje de Cáncer de Mama (11). En todas se ha realizado un análisis combinado de los resultados de nueve ensayos clínicos aleatorizados (ECA) en los que se incluyeron más de 600000 mujeres entre 39 y 74 años ${ }^{(28-36)}$ (Tabla 2).

\section{EFICACIA DEL TAMIZAJE MAMOGRÁFICO SOBRE LA MORTALIDAD}

La RS de Gøtzsche (24) reportó una disminución relativa de la mortalidad del 10 y $25 \%$ en los ECA, con una aleatorización óptima y subóptima, respectivamente. Esta RS concluye que una estimación más rigurosa del efecto sería del $15 \%$, punto intermedio entre 10 y $25 \%$. Por tanto, en términos absolutos, se evitaría una muerte de cada 2000 mujeres invitadas al tamizaje durante 10 años (Tabla 3).

A diferencia de la RS de Gøtzsche que utilizó para el metanálisis un modelo de efecto fijo, la RS publicada por el Grupo del Reino Unido ${ }^{(25)}$ utilizó uno de efecto aleatorio. La principal diferencia consiste en que el modelo de efectos aleatorios incorpora en el análisis la variabilidad entre los estudios, lo que mejoraría el nivel de confianza y, en cierto modo, la validez externa de los resultados ${ }^{(37)}$. Con este modelo se reportó una disminución relativa de la mortalidad del $20 \%$. Aplicando este porcentaje a la realidad británica, donde las mujeres entre 50 y 69 años participan en el tamizaje de forma trienal, los autores estiman que para evitar una muerte sería necesario invitar a 235 mujeres durante 20 años.

Por su parte, los grupos canadiense y estadounidense $(26,38)$ identificaron el mayor beneficio en el grupo de 50 a 69 años $(21 \%)$ reportando que para evitar una muerte sería 
Tabla 3. Beneficios y riesgos del cribado mamográfico. Resultados de las principales RS publicadas entre el 2009 y 2013.

\begin{tabular}{|c|c|c|c|c|c|c|c|c|c|}
\hline \multirow{2}{*}{$\begin{array}{l}\text { Revisión } \\
\text { Sistemática } \\
\text { (referencia) }\end{array}$} & \multicolumn{4}{|c|}{ Disminución de la mortalidad } & \multicolumn{5}{|c|}{ Riesgos } \\
\hline & $\begin{array}{c}\text { Número } \\
\text { de } \\
\text { estudios }\end{array}$ & $\begin{array}{l}\text { Análisis de } \\
\text { subgrupo }\end{array}$ & $\begin{array}{l}\text { Relativa* } \\
\text { (IC 95\%) }\end{array}$ & $\begin{array}{l}\text { Absolutaף } \\
\text { (intervalo del } \\
\text { cribado) }\end{array}$ & $\begin{array}{l}\text { Número } \\
\text { de } \\
\text { estudios }\end{array}$ & $\begin{array}{l}\text { Periodo } \\
\text { evaluado }\end{array}$ & $\begin{array}{l}\text { Falsos } \\
\text { positivos }\end{array}$ & $\begin{array}{l}\text { Exceso de } \\
\text { procedimientos } \\
\text { invasivos }\end{array}$ & $\begin{array}{l}\text { Sobrediag- } \\
\text { nóstico }\end{array}$ \\
\hline \multirow{3}{*}{$\begin{array}{l}\text { Cochrane } \\
\text { Reviews }^{23}\end{array}$} & 8 & $\begin{array}{l}\text { Estimación } \\
\text { final }\end{array}$ & $\sim 15 \%$ & \multirow{3}{*}{$\begin{array}{c}1 / 2000 \text { invitadas } \\
\text { durante } 10 \text { años } \\
(B / T)\end{array}$} & \multirow{3}{*}{3} & \multirow{3}{*}{$\begin{array}{l}\text { acumulado } \\
13 \text { años }\end{array}$} & \multirow{3}{*}{$49 \%$} & \multirow{3}{*}{$19 \%$} & \multirow{3}{*}{$30 \%$} \\
\hline & 3 & $\begin{array}{l}\text { Adecuada } \\
\text { aleatorización }\end{array}$ & $\begin{array}{c}10 \% \\
(-2 \text { a } 21)\end{array}$ & & & & & & \\
\hline & 5 & $\begin{array}{l}\text { Inadecuada } \\
\text { aleatorización }\end{array}$ & $\begin{array}{c}25 \% \\
(17 \text { a } 33)\end{array}$ & & & & & & \\
\hline $\begin{array}{l}\text { UK National } \\
\text { Health } \\
\text { Service }^{24}\end{array}$ & 8 & & $\begin{array}{c}20 \% \\
(11 \mathrm{a} 27)\end{array}$ & $\begin{array}{c}1 / 235 \text { invitadas } \\
\text { durante } 20 \text { años }(\mathrm{T})\end{array}$ & 3 & $\begin{array}{l}\text { tras una } \\
\text { mamografía }\end{array}$ & $3,2 \%$ & $1 \%$ & $11-19 \%$ \\
\hline \multirow{3}{*}{$\begin{array}{l}\text { Canadian } \\
\text { TFPHC }^{25}\end{array}$} & 8 & 39 a 49 años & $\begin{array}{c}15 \% \\
(4 \text { a } 25)\end{array}$ & $\begin{array}{c}1 / 2108 \text { invitadas } \\
\text { durante } 11 \text { años }(\mathrm{B} / \mathrm{T})\end{array}$ & 8 & $\begin{array}{l}\text { acumulado } \\
11 \text { años }\end{array}$ & $33 \%$ & $3,6 \%$ & $30-52 \%$ \\
\hline & 7 & 50 a 69 años & $\begin{array}{c}21 \% \\
(10 \mathrm{a} 32)\end{array}$ & $\begin{array}{c}1 / 720 \text { invitadas } \\
\text { durante } 11 \text { años }(\mathrm{B} / \mathrm{T})\end{array}$ & 7 & $\begin{array}{l}\text { acumulado } \\
11 \text { años }\end{array}$ & $28 \%$ & $3,7 \%$ & NR \\
\hline & 2 & 70 a 74 años & $\begin{array}{c}32 \% \\
(-1 \text { a } 55)\end{array}$ & No reportado & 2 & $\begin{array}{l}\text { acumulado } \\
11 \text { años }\end{array}$ & $21 \%$ & $2,6 \%$ & NR \\
\hline \multirow{4}{*}{$\begin{array}{l}\text { US PSTF } \\
2009^{26}\end{array}$} & 8 & 39 a 49 años & $\begin{array}{c}15 \% \\
(4 \text { a } 25)\end{array}$ & $\begin{array}{c}\text { 1/1 } 904 \text { invitadas } \\
\text { durante } 12 \text { años }(\mathrm{B} / \mathrm{T})\end{array}$ & 8 & $\begin{array}{l}\text { tras una } \\
\text { mamografía }\end{array}$ & $10 \%$ & NR & $1-10 \%$ \\
\hline & 6 & 50 a 59 años & $\begin{array}{c}24 \% \\
(1 \text { a } 25)\end{array}$ & $\begin{array}{c}1 / 1339 \text { invitadas } \\
\text { durante } 12 \text { años }(\mathrm{B} / \mathrm{T})\end{array}$ & 6 & $\begin{array}{l}\text { tras una } \\
\text { mamografía }\end{array}$ & $11 \%$ & NR & $1-10 \%$ \\
\hline & 2 & 60 a 69 años & $\begin{array}{c}32 \% \\
(10 \text { a } 32)\end{array}$ & $\begin{array}{c}\text { 1/337 invitadas } \\
\text { durante } 7 \text { años }(\mathrm{B} / \mathrm{T})\end{array}$ & 2 & $\begin{array}{l}\text { tras una } \\
\text { mamografía }\end{array}$ & $14 \%$ & NR & $1-10 \%$ \\
\hline & 1 & 70 a 74 años & $\begin{array}{c}-12 \% \\
(-72 \text { a } 27)\end{array}$ & $\begin{array}{l}\text { No es posible realizar } \\
\text { el calculo }\end{array}$ & 1 & $\begin{array}{l}\text { tras una } \\
\text { mamografía }\end{array}$ & $15 \%$ & NR & $1-10 \%$ \\
\hline \multirow{2}{*}{$\begin{array}{l}\text { Euroscreen } \\
\text { Working } \\
\text { Group }^{10}\end{array}$} & 7 & IBM & $\begin{array}{c}25 \% \\
(19 \text { a } 31)\end{array}$ & \multirow{2}{*}{$\begin{array}{c}7-9 / 1000 \text { cribadas } \\
\text { durante } 20 \text { años y } \\
\text { seguidas hasta los } 79 \\
\text { años (B) }\end{array}$} & $\begin{array}{l}\text { con ajuste } \\
\text { adecuado }\end{array}$ & \multirow{2}{*}{$\begin{array}{l}\text { acumulado } \\
20 \text { años }\end{array}$} & \multirow{2}{*}{$20 \%$} & \multirow{2}{*}{$3 \%$} & $0-10 \%$ \\
\hline & 8 & $\begin{array}{l}\text { Casos y } \\
\text { controles }\end{array}$ & $\begin{array}{c}31 \% \\
(27 \text { a } 43)\end{array}$ & & $\begin{array}{l}\text { estudios } \\
\text { sin ajuste }\end{array}$ & & & & $1-58 \%$ \\
\hline
\end{tabular}

$\mathrm{RS}=$ revisiones sistemáticas, $\mathrm{NR}=$ no reportado, $\mathrm{IBM}=$ incidence-based mortality (estudios que midieron las mortalidad en mujeres que con certeza se realizaron una o más pruebas de cribado mamográfico.

*T La disminución relativa y la absoluta se obtienen dimensionando la diferencia entre el número de muertes que se producen en el grupo de mujeres invitadas al cribado frente al grupo control. Mientras que la disminución relativa, es la proporción o porcentaje en que se reduce el riesgo relativo de muerte por cáncer de mama, la disminución absoluta se expresa mediante el número total de muertes evitadas.

necesario invitar a participar durante 11 años a 720 mujeres (Tabla 3).

Si bien la mejor forma de medir la eficacia de una intervención es mediante los estudios experimentales, una aproximación más cercana a la realidad podría obtenerse a partir de otros diseños pragmáticos ${ }^{(39)}$. Bajo esta premisa, el grupo europeo Euroscreen realizó un metanálisis de estudios observacionales. Además de los estudios de tipo caso control, se incluyeron aquellos que siguieron una metodología incidence-based mortality (IBM), lo que permitiría un manejo más adecuado del factor exposición (40). La disminución en la mortalidad estimada a partir de los estudios IBM fue del $25 \%$ y del $31 \%$ en los estudios de tipo caso control. Así, los autores reportaron que realizando el tamizaje bienal a 1000 mujeres durante 20 años se evitarían 7 a 9 muertes ${ }^{(11)}$ (Tabla 3).

\section{SEGURIDADDELTAMIZAJE.ELSOBREDIAGNÓSTICO}

Los estudios disponibles dimensionaron el sobrediagnóstico como el exceso de neoplasias detectadas en el grupo tamizaje frente al número total de neoplasias clínicamente diagnosticadas en el grupo control. Dos perspectivas, la poblacional y la individual, han sido utilizadas para reportar el sobrediagnóstico. En ambas, el numerador está determinado por el exceso de neoplasias. Bajo una perspectiva individual, en el denominador se incluirían solo las neoplasias diagnosticadas durante el periodo del tamizaje mientras que bajo una perspectiva poblacional se incluirian también las neoplasias diagnosticadas durante el periodo posterior al tamizaje.

Tanto la RS Cochrane ${ }^{(24)}$ como la del Reino Unido ${ }^{(25)}$ consideraron adecuados para el estudio del sobrediagnóstico a tres ECA ${ }^{(28,29,41)}$. En estos, las mujeres aleatorizadas al grupo control no fueron invitadas a participar de ningún tamizaje posterior a la intervención y tuvieron un periodo de seguimiento adecuado. La frecuencia de sobrediagnóstico fue del orden del $11 \%$ desde una perspectiva poblacional y alrededor del $19 \%$ desde la individual ${ }^{25)}$. Según la RS Cochrane esta cifra sería mayor, cercana al 30\%, 
debido al tamizaje oportunista en el grupo control. Cifras menores (1 a 10\%) se reportaron a partir de los estudios observacionales ${ }^{(38,42)}$. Con estos datos, los autores estimaron que de cada 1000 mujeres invitadas a participar del tamizaje bienal durante 20 años, entre 4 y 20 mujeres sufrirían un sobrediagnóstico (11,24). Dicho de otra manera, si una mujer cribada recibe el diagnóstico de cáncer, la probabilidad de que se trate de un sobrediagnóstico sería de un 19\% ${ }^{(25)}$ (Tabla 3).

\section{EVALUACIÓN DE LA CALIDAD DE LA EVIDENCIA}

La mayoría de los EC incluidos en las RS mostraron limitaciones serias con posibles distorsiones en la aleatorización, ocultamiento de la secuencia, y cegamiento. Además, todos estos ECA fueron realizados hace aproximadamente 20 o 30 años por lo que su validez hoy en día podría quedar en entredicho, más aun considerando que el riesgo basal de la población femenina peruana es diferente a la población europea. Sin embargo, los resultados fueron consistentes, se midieron de forma directa y mostraron una gran precisión, por lo que finalmente la calidad de la evidencia fue moderada (Tabla 4).
El escenario es diferente para los estudios observacionales. En este caso, a pesar de tratarse de estudios contemporáneos, la calidad de la evidencia podría ser de baja o muy baja calidad debido a las limitaciones propias de su diseño, medición errónea entre la exposición y el desenlace, problemas con el manejo de las variables de confusión y grupos de comparación inadecuados (Tabla 5).

\section{BALANCE ENTRE LOS RIESGOS Y BENEFICIOS}

La incertidumbre en torno a las estimaciones del beneficio y el sobrediagnóstico fueron importantes. Por cada 1000 mujeres que participen del tamizaje durante 20 años, entre 2 y 9 muertes serían evitadas y de 4 a 20 mujeres sufrirían un sobrediagnóstico. Los principales determinantes de esta gran variabilidad fueron el tipo de estudio, la edad de las mujeres, la duración del tamizaje y del seguimiento. En todo caso, aunque el número de casos sobrediagnosticados parece ser más frecuente que el número absoluto de muertes evitadas, una clave para considerar un balance negativo sería determinar si las consecuencias del sobrediagnóstico alcanzan una magnitud cercana a la muerte.

Tabla 4. Resumen de la evidencia y evaluación de la calidad de los ensayos clínicos aleatorizados (ECA) que evaluaron el efecto del cribado mamográfico sobre la mortalidad por cáncer de mama. Adaptado de la Guía de Práctica Clínica (GPC) Nacional Canadiense ${ }^{26}$.

\begin{tabular}{|c|c|c|c|c|c|c|c|c|c|c|c|}
\hline \multirow[b]{3}{*}{$\begin{array}{l}\text { Número de } \\
\text { estudios }\end{array}$} & \multirow[b]{3}{*}{ Diseño } & \multirow{2}{*}{\multicolumn{3}{|c|}{ Evaluación de la calidad }} & \multirow[b]{3}{*}{ Imprecisión } & \multirow[b]{3}{*}{ Otros } & \multicolumn{4}{|c|}{ Resumen de los resultados } & \multirow[b]{3}{*}{$\begin{array}{l}\text { Calidad de } \\
\text { la evidencia }\end{array}$} \\
\hline & & & & & & & \multicolumn{2}{|c|}{$\begin{array}{c}\text { Mortalidad } \\
\text { por cáncer de } \\
\text { mama }\end{array}$} & \multicolumn{2}{|r|}{ Efecto } & \\
\hline & & $\begin{array}{l}\text { Limitaciones } \\
\text { en el estudio }\end{array}$ & Inconsistencia & $\begin{array}{l}\text { Evidencia } \\
\text { indirecta }\end{array}$ & & & $\begin{array}{c}\text { Cribado } \\
\mathrm{n}(\%)\end{array}$ & $\begin{array}{c}\text { Control } \\
\mathrm{n}(\%)\end{array}$ & $\begin{array}{c}\mathrm{RR} \\
\text { (IC } 95 \%)\end{array}$ & Absoluto (NNC) & \\
\hline \multicolumn{12}{|c|}{ Mortalidad por cáncer de mama en mujeres de 40 a 49 años } \\
\hline 8 & ECA & Serio† & No serio§ & No serioף & No serio** & Not† & $\begin{array}{c}448 \\
(0,29)\end{array}$ & $\begin{array}{c}625 \\
(0,32)\end{array}$ & $\begin{array}{c}0,85 \\
(0,75-0,96)\end{array}$ & $\begin{array}{l}1 \text { por cada } 2108 \\
\text { ) cribadas } 11 \text { años }\end{array}$ & $\begin{array}{l}8 \text { Moderada } \\
\text { s }\end{array}$ \\
\hline \multicolumn{12}{|c|}{ Mortalidad por cáncer de mama en mujeres de 50 a 69 años } \\
\hline 7 & ECA & Serioł‡ & No serio§§ & No seriođ & No serio** & Not† & $\begin{array}{c}639 \\
(0,47)\end{array}$ & $\begin{array}{c}743 \\
(0,64)\end{array}$ & $\begin{array}{c}0,79 \\
(0,68-0,90)\end{array}$ & $\begin{array}{c}1 \text { por cada } 721 \\
\text { ) cribadas } 11 \text { años }\end{array}$ & s Moderada \\
\hline \multicolumn{12}{|c|}{ Mortalidad por cáncer de mama en mujeres de 70 a 74 años } \\
\hline 2 & ECA & Serio ${ }^{* * *}$ & No seriot†† & No seriođ & 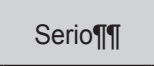 & Not† & $\begin{array}{c}49 \\
(0,47)\end{array}$ & $\begin{array}{c}50 \\
(0,68)\end{array}$ & $\begin{array}{c}0,68 \\
(0,45-1,01)\end{array}$ & NE & Baja \\
\hline
\end{tabular}

Nota: Las estimaciones de RR (riesgo relativo) se basan en el meta-análisis con modelo de efectos aleatorios..

$\mathrm{ECA}=$ ensayo clínico aleatorizado. $\mathrm{RR}=$ riesgo relativo. $\mathrm{IC}=$ intervalo de confianza. NNC = número necesario a cribar (mujeres que necesitan ser cribadas cada 2 años durante un promedio de 11 años para prevenir 1 muerte por cáncer de mama). NE= No estimable debido a la falta de precisión del RR, la intervención podría incrementar la mortalidad en lugar de reducirla.

† Cinco estudios cuasi-aleatorizados y tres ECA verdaderos. El cegamiento y el ocultamiento de la secuencia no están claros en los cinco primeros.

$\S$ No existe heterogeneidad, $p=0,48 ; 1^{2}=0 \%$.

I La evidencia que se evalúa es directa. El efecto del cribado sobre la reducción de la mortalidad es una pregunta que se aborda de forma similar en

todos los estudios en relación a la población, intervención, comparación y resultados.

** El tamaño total de la muestra es grande y IC95\% de los resultados es estrecho.

†† No se cuenta con estudios suficientes para evaluar el sesgo de publicación.

拉 El cegamiento y el ocultamiento de la secuencia no están claros en los cinco estudios y sólo dos son ECA verdaderos.

$\S \S$ No existe heterogeneidad, $p=0,12,\left.\right|^{2}=41 \%$.

${ }_{* \star *}$ El cegamiento y ocultamiento no están claros.

††† No existe heterogeneidad, $p=0,75 ; \imath^{2}=0 \%$.

ๆๆ El tamaño total de la muestra es grande, pero el número total de eventos es pequeño lo que condiciona un IC95\% amplio. 
Tabla 5. Tabla GRADE de los estudios observacionales que evaluaron el efecto del cribado mamográfico sobre la mortalidad por cáncer de mama. Elaboración propia a partir de los estudios incluidos en la Revisión Sistemática (RS) del Grupo Europeo Euroscreen ${ }^{11}$.

\begin{tabular}{|c|c|c|c|c|c|c|c|c|c|c|c|}
\hline \multirow[b]{3}{*}{$\begin{array}{l}\text { Número de } \\
\text { estudios }\end{array}$} & \multirow[b]{3}{*}{ Diseño } & \multirow{2}{*}{\multicolumn{3}{|c|}{ Evaluación de la calidad }} & \multirow[b]{3}{*}{ Imprecisión } & \multirow[b]{3}{*}{ Otros } & \multicolumn{4}{|c|}{ Resumen de los resultados } & \multirow[b]{3}{*}{$\begin{array}{l}\text { Calidad de } \\
\text { la evidencia }\end{array}$} \\
\hline & & & & & & & \multicolumn{2}{|c|}{$\begin{array}{l}\text { Mortalidad por } \\
\text { cáncer de mama }\end{array}$} & \multicolumn{2}{|c|}{ Efecto } & \\
\hline & & $\begin{array}{l}\text { Limitaciones } \\
\text { en el estudio }\end{array}$ & Inconsistencia & $\begin{array}{l}\text { Evidencia } \\
\text { indirecta }\end{array}$ & & & $\begin{array}{c}\text { Invitadas al } \\
\text { cribado } \\
\mathrm{n}(\%)\end{array}$ & $\begin{array}{c}\text { No } \\
\text { invitadas } \\
\mathrm{n}(\%)\end{array}$ & $\begin{array}{l}\text { Relativo } \\
\text { (IC 95\%) }\end{array}$ & $\begin{array}{l}\text { Absoluto } \\
\text { (NNC) }\end{array}$ & \\
\hline \multicolumn{12}{|c|}{ Mortalidad por cáncer de mama en mujeres de 45 a 69 años } \\
\hline 20 & IBM & No serioł & No serio§ & No seriođ & No serio* & No† & ND & ND & $\begin{array}{c}\text { RR } 0,75 \\
(0,69-0,81)\end{array}$ & $N D^{* * *}$ & Baja \\
\hline \multicolumn{12}{|c|}{ Mortalidad por cáncer de mama en mujeres de 45 a 69 años (cribado) frente a otros grupos de edad ( 25 a 85 , control) } \\
\hline 12 & $\begin{array}{l}\text { Estudio de } \\
\text { tendencia }\end{array}$ & Serioł‡ & Serio§§ & SerioףT & No serio** & Not & ND & ND & $\begin{array}{c}\text { Reducción } \\
\text { anual†† } \\
1-9 \% \\
\text { Reducción al } \\
\text { finaltt† } \\
28-36 \%\end{array}$ & ND & Muy baja \\
\hline \multicolumn{12}{|c|}{ Mortalidad por cáncer de mama en mujeres de 40 a 79 años } \\
\hline 7 & $\mathrm{CC}$ & Seriołł & No serio§§§ & No seriođ & No serio* & No† & ND & ND & $\begin{array}{c}\text { OR } 0,69 \\
(0,57-0,73) \\
\end{array}$ & $N D^{* * *}$ & Muy baja \\
\hline
\end{tabular}

Nota: Los estudios observacionales presentan serias limitaciones para estimar el efecto de una intervención. De acuerdo al sistema GRADE la calidad de la evidencia derivada de estudios observacionales es baja. Podría mejorar si se demuestra un adecuado manejo de las variables de confusión, una gran magnitud del efecto y un gradiente dosis-respuesta.

IBM= estudios que siguen la metodología incidence-based mortality. Los eventos se miden solo en aquellas mujeres que fueron diagnosticadas de cáncer de mama después de su primera invitación al cribado. $\mathrm{CC}=$ estudio caso control. $\mathrm{IC}=$ intervalo de confianza. $\mathrm{RR}=$ riesgo relativo. $\mathrm{OR}=$ odds ratio. $\mathrm{NNC}=$ número necesario a cribar ND= no disponible.

‡ Manejo adecuado de las variables de confusión más relevantes. El diseño permite reducir el riesgo de errores en la medición de la exposición a la intervención y el desenlace. El grupo control mostró características similares al grumo intervención en edad y localización geográfica. La magnitud del efecto es importante. No se cuenta con información sobre el gradiente dosis-respuesta.

$\S$ No existe heterogeneidad, $p=0,40,1^{2}=$ no reportado

T El efecto del cribado sobre la mortalidad se mide de forma directa. El resultado se mide posterior a la intervención, no se evalúan resultados intermedios, la población utilizada y los instrumentos de medición son apropiados para evaluar el efecto.

${ }^{*}$ El tamaño de la muestra es adecuado y el IC $95 \%$ es estrecho.

† No se evaluó el sesgo de publicación.

†† Reducción de la mortalidad reportada de forma anual.

†† Reducción de la mortalidad reportada al final del periodo del cribado.

拉 No se reportó un manejo adecuado de las variables de confusión. Sólo se ajustó el sesgo de auto-selección. El riesgo de sesgo es alto.

$\S \S$ Gran heterogeneidad. Los autores no realizaron un análisis combinado de los datos debido a la gran variabilidad entre los estudios (instrumentos de medición, grupos de comparación).

ๆTा Importantes diferencias epidemiológicas entre los grupos de comparación. La incidencia de cáncer de mama varía de acuerdo a la edad siendo más frecuente en mujeres de 45 a 69 años.

** El tamaño total de la muestra es grande. No se cuenta con el IC95\% de las estimaciones.

$\S \S \S$ No existe heterogeneidad, $p=0,17 ; 1^{2}=$ no reportado.

${ }^{* * *}$ En un estudio posterior, considerando solo los datos de los estudios IBM y CC, los autores estiman que para reducir la mortalidad en 7 a 9 casos es necesario cribar a 1000 mujeres $^{18}$

La magnitud del efecto del sobrediagnóstico es difícil de calcular, en parte, porque no se ha podido cuantificar claramente su frecuencia. Es difícil determinar si una imagen sospechosa representa realmente un peligro para la mujer o, por el contrario, se trata de una lesión que nunca afectará su vida. Como ejemplo, los autores han considerado al carcinoma ductal in situ. Debido a su progresión lenta o estacionaria, en muchos casos este carcinoma no progresaría a estadios avanzados. Si fuera identificado en el tamizaje, sería innecesariamente tratado afectando negativamente la calidad de vida y el bienestar psicológico y económico de la mujer ${ }^{(15)}$.

Otra clave para orientar bien el balance entre los riesgos y beneficios es identificar los grupos que más se beneficiarían de la intervención. Consistentemente el grupo de edad en que se observan los mayores beneficios es el de 50 a 69 años. Mujeres de otras edades podrían estar igual de expuestas a un sobrediagnóstico y obtendrían menos beneficios del tamizaje mamográfico.

\section{USO DE LOS RECURSOS}

Se han identificado dos evaluaciones económicas que comparan diferentes estrategias para el control del cáncer de mama en el Perú ${ }^{(43,44)}$. Ambos estudios coinciden en reportar que solo brindar tratamiento a los casos sintomáticos sin incorporar ningún tipo de prevención sería la estrategia menos recomendable. 
En el estudio de costo-efectividad de Zelle et al. ${ }^{(43)}$, se comparan 94 posibles escenarios que combinan las actividades de prevención y tratamiento más relevantes en Perú. El análisis se realizó desde la perspectiva del Sistema de Salud y siguiendo la metodología recomendada por la OMS para el desarrollo de evaluaciones económicas de países en vías de desarrollo (WHO-CHOICE) ${ }^{(45)}$. Se consideraron muy costo-efectivas a las intervenciones con una relación de costo-efectividad incremental (RCE) por debajo de \$ 4608 para evitar un AVISA. El tamizaje con mamografía trienal en mujeres de 45 a 69 años, $60 \%$ fija (urbana) y $40 \%$ móvil (rural) combinado con el tratamiento estándar, sin trastuzumab, en todos los estadios de la enfermedad mostró una RCE de \$ 4125 por AVISA, siendo así la estrategia más costoefectiva. Ampliar el rango del tamizaje trienal hasta los 40 años y realizarlo de forma bienal incrementaría la RCE a $\$ 5659$ y $\$ 27477$, respectivamente. Otras estrategias, incluidas las combinaciones con examen clínico y punción inmediata con aguja fina (PAF), mostraron RCE por encima del umbral recomendado ${ }^{(43)}$.

Desde la misma perspectiva del sistema de salud, un estudio de costo-utilidad comparó los años de vida ajustados por calidad (AVAC) que se obtendrían a partir de cinco diferentes estrategias. La mamografía, con un promedio en costo y AVAC de $\$ 35,93$ y $\$ 0,3963$, respectivamente, se reportó como la estrategia más recomendable. Tal como refiere el autor, la incertidumbre alrededor de esta estimación es bastante amplia, debido principalmente a la falta de datos representativos de la población peruana ${ }^{(44)}$.

\section{VALORES Y PREFERENCIAS}

En Perú los estudios sobre conocimientos, actitudes, valores o preferencias de las mujeres frente al tamizaje de cáncer de mama son escasos ${ }^{(46-48)}$. Recientemente Huaman et al. realizaron un estudio de validación del cuestionario de susceptibilidad, beneficios y barreras ante el tamizaje mamográfico en 296 mujeres entre 40 y 65 años. El 57,2\% de las participantes refirió haberse realizado una mamografía alguna vez en su vida. En el análisis multivariado, los únicos factores asociados a realizarse una mamografía fueron la edad entre 50 y 60 años y tener conocimientos medios o altos sobre la mamografía y el cáncer de mama. Por el contrario, la percepción de las barreras (miedo, vergüenza, olvido, priorización de otras necesidades, etc.) estuvo fuertemente asociada a la no realización de la mamografía ${ }^{(46)}$.

Aunque la utilidad del tamizaje parece ser reconocida en la población femenina peruana esto no queda reflejado en la realización habitual de este tipo de pruebas. Un estudio nacional en base a la Encuesta Demográfica de Salud Familiar 2008 (ENDES) reportó que un 34,6\% de las mujeres entre 20 y 49 años se había realizado alguna vez el autoexamen de mamas. La realización de la mamografía sería mucho menos frecuente y con importantes diferencias geográficas a nivel nacional ${ }^{(49)}$.

\section{RECOMENDACIÓN FINAL}

Como se muestra en la Tabla 6, basados en el análisis del equilibrio entre beneficios, riesgos, costos y preferencias, la implementación del tamizaje mamográfico en Perú sería débilmente recomendable en el grupo de mujeres de 50 a 69 años. Por otro lado, esta intervención no sería recomendable en las mujeres menores de 50 y mayores de 69 años debido a que los beneficios esperados no superarían los riesgos del tamizaje.

\section{DISCUSIÓN}

En el Perú las estrategias que no incorporan medidas preventivas y solo se basan en el tratamiento, han demostrado ser las menos recomendables ${ }^{(43,44)}$. Es innegable el impacto negativo que esta enfermedad tiene en la sociedad por lo que de forma muy acertada se han iniciado importantes esfuerzos para mejorar el manejo del cáncer a nivel nacional. En todos los contextos los recursos son siempre limitados y aquellos que se dediquen al control de una enfermedad no pueden dedicarse a otras actividades. Este costo de oportunidad nos obliga a evaluar muy rigurosamente las estrategias disponibles y elegir las más eficientes.

Particularmente, en relación con el cáncer de mama, la propuesta de la Coalición Multisectorial consiste en realizar el tamizaje a las mujeres entre los 40 y 69 años, de preferencia se realizará examen clínico asociado a PAF en el grupo de 40 a 49 y mamográfico a partir de los $50{ }^{(15)}$. Algunas consideraciones alrededor de esta propuesta podrían mejorar los resultados de una intervención a gran escala.

Sobre el tamizaje en el grupo de 40 a 49 años. No existe evidencia a favor del examen clínico como prueba de tamizaje a nivel poblacional, no ha demostrado reducir la mortalidad ni mejorar la detección del cáncer en estadios tempranos. De hecho, independientemente de la edad de la mujer, la OMS solo recomienda el tamizaje clínico en lugares en los que la mamografía no está disponible ${ }^{(50)}$. Además, en mujeres jóvenes la frecuencia de la enfermedad no es alta, lo que reduciría aun más el valor predictivo positivo del examen incrementando el número de lesiones benignas detectadas. En este 


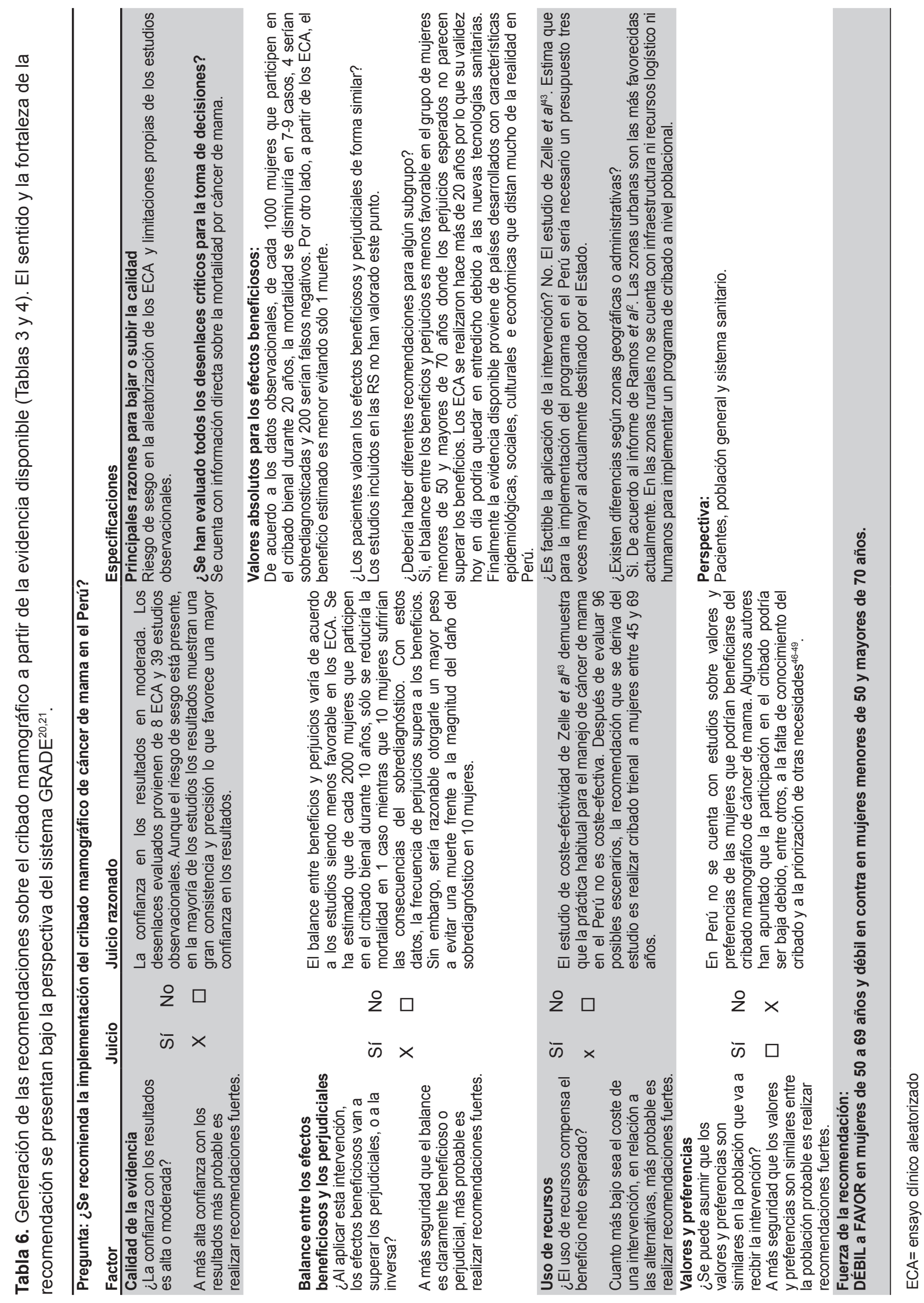


contexto, realizar una prueba invasiva, como es el PAF, para determinar la histología del tumor podría mejorar la precisión en los resultados. En contraposición, la PAF incrementaría los costos y la necesidad de recursos humanos adecuadamente entrenados. Por tanto, si incorporamos todos estos factores bajo la perspectiva GRADE, la recomendación sería débil en contra de este tipo de tamizaje en mujeres de 40 a 59 años.

En las mujeres a partir de los 50 años el tamizaje mamográfico trienal parece ser la prueba de elección. Reducir el intervalo a bienal o anual incrementaría los costos sin mejorar sensiblemente los beneficios, lo que representaría una mala utilización de los recursos (43). No solo es suficiente conocer el perfil de costoefectividad o costo-utilidad de una intervención antes de implementarla, también es necesario valorar el costo de la información que será necesaria para evaluar los resultados de la misma ${ }^{(51)}$. En Perú, este valor de la información podría ser alto. Sería necesario, por ejemplo, implementar un registro que permita evaluar cambios en los estadios al diagnóstico y su repercusión en la mortalidad. Así también tendrán que generarse otras fuentes de información para valorar el cumplimiento de los estándares del tamizaje.

Por otro lado, la participación y adherencia de las mujeres depende en gran medida de sus conocimientos y actitudes. En México, la baja participación reportada $(22 \%)^{(52)}$ sería parcialmente atribuible a la escasa información, la incomodidad y el miedo que sienten las mujeres frente al tamizaje ${ }^{(53)}$. En Perú, una gran área de trabajo consistirá en educar a la población y mejorar la confianza de las mujeres en los servicios de salud. Según los estudios disponibles si los porcentajes de participación no acercan al $70 \%$ la intervención reduciría su nivel de costo-efectividad y los recursos asignados serían difícilmente justificables ${ }^{(43)}$.

Finalmente, una barrera frente a la implementación de un programa coordinado a nivel nacional es la actual organización segmentada del sistema sanitario. El 85\% de los médicos oncólogos ejercen en Lima donde, además, se concentran la mayoría de los equipos de prevención, diagnóstico y tratamiento del cáncer ${ }^{(54)}$. Del mismo modo, la disponibilidad al tratamiento, que es un componente crucial en el manejo del cáncer, no está garantizada en todo el territorio nacional. Las poblaciones rurales estarían especialmente vulnerables debido a que los centros locales no pueden ofrecer un adecuado cuidado de las pacientes con cáncer. Por tanto, un reto importante consistirá en el equipamiento de los servicios de salud priorizando la máxima utilización de los recursos humanos y logísticos disponibles.

\section{CONCLUSIONES}

El tamizaje de cáncer de mama no solo supone beneficios sino también riesgos para la población participante. Si bien la mamografía es la única estrategia que ha demostrado una reducción en la mortalidad, el balance entre los riesgos y beneficios podría ser negativo si la intervención se implementa en la población equivocada. En Perú la recomendación es débil a favor del tamizaje mamográfico en las mujeres de 50 a 69 años y débil en contra en las mujeres fuera de este rango de edad. La evidencia disponible indica que los resultados más beneficiosos y costo-efectivos se presentarían mediante un tamizaje trienal en mujeres de 50 a 69 años. En lugares donde la mamografía está disponible, el tamizaje clínico no parece justificarse, mucho menos en mujeres jóvenes en quienes la prevalencia de la enfermedad no es alta.

Importantes retos en materia de equidad, educación, redistribución de los recursos, acceso y mejora de la confianza en los sistemas sanitarios podrían condicionar los resultados del tamizaje a nivel nacional. Frente a otras posibles áreas en las que invertir los recursos disponibles, solo mediante la evaluación rigurosa de los resultados del programa se podrá valorar si es justificable continuar con la intervención elegida. La toma de decisiones basadas en la evidencia es indispensable, en este sentido el sistema GRADE es una herramienta de gran utilidad.

Contribuciones de autoría: XBC y MPR han participado en la concepción del artículo, y su redacción. La búsqueda sistemática de la literatura, así como el análisis e interpretación de los datos fueron realizados por MPR y supervisados por XBC y TPR. TPR y XBC realizaron la revisión crítica del artículo $\mathrm{y}$ todos los autores han aprobado su versión final..

Conflictos de interés: los autores declaran no tener conflictos de interés.

Fuentes de financiamiento: autofinanciado. 


\section{REFERENCIAS BIBLIOGRÁFICAS}

1. Ferlay J, Soerjomataram I, Ervik M, Dikshit R, Eser S, Mathers C, et al. GLOBOCAN 2012: Estimated cancer incidence, mortality and prevalence worldwide in 2012 [Internet]. Lyon, France: International Agency for Research on Cancer; 2013 [citado el 22 de mayo del 2014]. Disponible en: http://globocan.iarc.fr

2. Ramos W, Venegas D. Análisis de la situación del cáncer en el Perú 2013. Lima: MINSA/DGE;2013.

3. Justo N, Wilking N, Jönsson B, Luciani $S$, Cazap E. A review of breast cancer care and outcomes in Latin America. Oncologist. 2013;18(3):248-56. doi: 10.1634/theoncologist.2012-0373.

4. Instituto Nacional de Estadística e Informática. Condiciones de vida en el Perú. Informe Técnico № 2. Lima: INEI; 2014.

5. Yip C-H, Cazap E, Anderson BO, Bright KL, Caleffi M, Cardoso F, et al. Breast cancer management in middle-resource countries (MRCs): consensus statement from the Breast Health Global Initiative. Breast. 2011 Apr;20 Suppl 2:S12-9. doi: 10.1016/j. breast.2011.02.015.

6. World Health Organization. Cribado y detección precoz del cáncer [Internet]. Geneva: WHO; c2015 [citado el 14 de enero 14 del 2015]. Disponible en: http://www.who.int/cancer/ detection/es/

7. Thomas D, Gao D, Ray R, Wang W, Allison C, Chen F, et al. Randomized trial of breast self-examination in Shanghai: final results. J Natl Cancer Inst. 2002 Oct 2;94(19):1445-57.

8. Nelson AL. Controversies regarding mammography, breast selfexamination, and clinical breast examination. Obstet Gynecol Clin North Am. 2013 Sep;40(3):413-27. doi: 10.1016/j.ogc.2013.05.001.

9. Spence D. Bad medicine: clinical breast examination. BMJ. 2012 Oct 11;345:e6789. doi: $10.1136 / \mathrm{bmj}$. e6789.

10. Puschel K, Thompson B. Mammogram screening in Chile: using mixed methods to implement health policy planning at the primary care level. Breast. 2011 Apr;20 Suppl 2:S40-5. doi: 10.1016/j.breast.2011.02.002.
11. Paci E, EUROSCREEN Working Group. Summary of the evidence of breast cancer service screening outcomes in Europe and first estimate of the benefit and harm balance sheet. J Med Screen. 2012;19 Suppl 1:5-13.

12. Broeders M, Moss S, Nyström L, Njor $S$, Jonsson H, Paap E, et al. The impact of mammographic screening on breast cancer mortality in Europe: a review of observational studies. J Med Screen. 2012;19 Suppl 1:14-25.

13. Giordano L, von Karsa L, Tomatis M, Majek O, de Wolf C, Lancucki L, et al. Mammographic screening programmes in Europe: organization, coverage and participation. J Med Screen. 2012;19 Suppl 1:72-82.

14. Perry N, Broeders M, de Wolf C, Törnberg S, Holland R, von Karsa L. European guidelines for quality assurance in breast cancer screening and diagnosis. Fourth edition-summary document. Ann Oncol. 2008 Apr;19(4):614-22.

15. Bond M, Pavey T, Welch K, Cooper C, Garside R, Dean S, et al. Systematic review of the psychological consequences of false-positive screening mammograms. Health Technol Assess. 2013 Mar;17(13):1-170, v-vi. doi: 10.3310/hta17130.

16. Salas D, Ibáñez J, Román R, Cuevas $\mathrm{D}$, Sala M, Ascunce N, et al. Effect of start age of breast cancer screening mammography on the risk of falsepositive results. Prev Med. 2011 JulAug;53(1-2):76-81. doi: 10.1016/j. ypmed.2011.04.013.

17. Marmot MG, Altman DG, Cameron DA, Dewar JA, Thompson SG, Wilcox M, et al. The benefits and harms of breast cancer screening: an independent review. $\mathrm{Br} \mathrm{J}$ Cancer. 2013 Jun 11;108(11):2205-40. doi: 10.1038/bjc.2013.177.

18. Jørgensen K. Mammography screening. Benefits, harms, and informed choice. Dan Med J. 2013 Apr;60(4):B4614.

19. Muir JA. Evidence-Based Healthcare: How to Make Health Policy and Management Decisions. Churchill Livingstone. New York; 2001.

20. Guyatt G, Oxman A, Akl E, Kunz R, Vist G, Brozek J, et al. GRADE guidelines: 1. Introduction-GRADE evidence profiles and summary of findings tables. J Clin Epidemiol. 2011 Apr;64(4):383-94. doi: 10.1016/j. jclinepi.2010.04.026.

21. GRADE Working Group [Internet]. c2005-2009 [citado el 14 de enero del 2015]. Disponible en: http://www. gradeworkinggroup.org/

22. Declaran de interés nacional la Atención Integral del Cáncer y Mejoramiento del Acceso a los Servicios Oncológicos en el Perú y dictan otras medidas. Decreto Supremo $N^{\circ}$ 009-2012-SA (3 de noviembre de 2012).

23. Instituto Nacional de Enfermedades Neoplasicas. Coalición Multisectorial "Perú contra el Cáncer". Plan Nacional para el Fortalecimiento de la Prevención y Control del Cáncer en el Perú. Lima: INEN; 2006.

24. Gøtzsche PC, Jørgensen KJ Screening for breast cancer with mammography. Cochrane Database Syst Rev. 2013 Jun 4;6:CD001877. doi: $10.1002 / 14651858 . C D 001877$. pub5.

25. Independent UK Panel on Breast Cancer Screening. The benefits and harms of breast cancer screening: an independent review. Lancet. $2012 \mathrm{Nov}$ 17;380(9855):1778-86. doi: 10.1016/ S0140-6736(12)61611-0.

26. The Canadian Task Force on Preventive Health Care. Recommendations on screening for breast cancer in averagerisk women aged 40-74 years. CMAJ. 2011 Nov 22;183(17):1991-2001. doi: 10.1503/cmaj.110334.

27. US Preventive Services Task Force Screening for breast cancer: U.S. Preventive Services Task Force Recommendation Statement. Ann Intern Med. 2009 Nov 17;151(10):71626, W-236. doi: 10.7326/0003-4819151-10-200911170-00008.

28. Miller AB, To T, Baines CJ, Wall C. The Canadian National Breast Screening Study-1: breast cancer mortality after 11 to 16 years of followup. A randomized screening trial of mammography in women age 40 to 49 years. Ann Intern Med. 2002 Sep 3;137(5 Part 1):305-12.

29. Miller AB, To T, Baines C-J, Wall C. Canadian National Breast Screening Study-2: 13-year results of a randomized trial in women aged 50 - 
59 years. J Natl Cancer Inst. 2000 Sep 20;92(18):1490-9.

30. Nyström L, Andersson I, Bjurstam N, Frisell J, Nordenskjöld B, Rutqvist LE. Long-term effects of mammography screening: updated overview of the Swedish randomised trials. Lancet. 2002 Mar 16;359(9310):909-19.

31. Tabar L, Gad A, Holmberg L, Ljungquist U. Significant reduction in advanced breast cancer. Results of the first seven years of mammography screening in Kopparberg, Sweden. Diagn Imaging Clin Med. 1985;54(34):158-64.

32. Andersson I, Aspegren K, Janzon L, Landberg T, Lindholm K, Linell F, et al. Mammographic screening and mortality from breast cancer: the Malmö mammographic screening trial. BMJ. 1988 Oct 15;297(6654):943-8.

33. Shapiro S, Venet W, Strax P, Venet L, Roeser R. Ten- to fourteen-year effect of screening on breast cancer mortality. J Natl Cancer Inst. 1982 Aug;69(2):349-55.

34. Fagerberg G, Baldetorp L, Gröntoft $\mathrm{O}$, Lundström $\mathrm{B}$, Månson JC, Nordenskjöld B. Effects of repeated mammographic screening on breast cancer stage distribution. Results from a randomised study of 92934 women in a Swedish county. Acta Radiol Oncol. 1985 Nov-Dec;24(6):465-73.

35. Frisell J, Lidbrink E, Hellström L, Rutqvist LE. Followup after 11 yearsupdate of mortality results in the Stockholm mammographic screening trial. Breast Cancer Res Treat. 1997 Sep;45(3):263-70.

36. Moss S, Cuckle H, Evans A, Johns L, Waller M, Bobrow L, et al. Effect of mammographic screening from age 40 years on breast cancer mortality at 10 years' follow-up: a randomised controlled trial. Lancet. $2006 \mathrm{Dec}$ 9;368(9552):2053-60.

37. Borenstein M, Hedges L, Higgins J, Rothstein $\mathrm{H}$. Introduction to MetaAnalysis. Chichester: John Wiley \& Sons.: 2009.

38. Nelson HD, Tyne K, Naik A, Bougatsos C, Chan BK, Humphrey L; U.S. Preventive Services Task Force. Screening for breast cancer: Systematic Evidence Review Update for the U.S. Preventive Services Task Force. Ann Intern Med.2009 Nov 17;151(10):727-
37, W237-42. doi: 10.7326/00034819-151-10-200911170-00009.

39. Ioannidis J, Greenland S, Hlatky MA, Khoury MJ. Increasing value and reducing waste in research design, conduct, and analysis. Lancet. 2014 Jan 11;383(9912):166-75. doi: 10.1016/ S0140-6736(13)62227-8.

40. Njor S, Nyström L, Moss S, Paci E, Broeders M, Segnan N, et al. Breast cancer mortality in mammographic screening in Europe: a review of incidence-based mortality studies. J Med Screen. 2012;19 Suppl 1:33-41.

41. Zackrisson S, Andersson I, Janzon L, Manjer J, Garne J. Rate of overdiagnosis of breast cancer 15 years after end of Malmo mammographic screening trial: follow-up study. BMJ. 2006 Mar 25;332(7543):689-92. Epub 2006 Mar 3.

42. Puliti D, Duffy S, Miccinesi G, de Koning H, Lynge E, Zappa M, et al. Overdiagnosis in mammographic screening for breast cancer in Europe: a literature review. J Med Screen. 2012;19 Suppl 1:42-56.

43. Zelle SG, Vidaurre T, Abugattas JE, Manrique JE, Sarria G, Jeronimo J, et al. Cost-effectiveness analysis of breast cancer control interventions in Peru. PLoS One. 2013 Dec 10;8(12):e82575. doi: 10.1371/journal.pone.0082575.

43. Gutiérrez-Aguado A. Costo utilidad de intervenciones preventivas para cáncer de mama en el Perú. Rev Peru Ginecol Obstet. 2012;58(4):253-61.

45. World Health Organization. Chosing interventions that are cost effective [Internet]. Geneva: WHO; c2015 [citado el 22 de mayo del 2014]. Disponible en: http://www.who.int/ choice/en/

46. Huaman MA, Kamimura-Nishimura KI, Kanamori M, Siu A, Lescano AG. Validation of a susceptibility, benefits, and barrier scale for mammography screening among Peruvian women: a cross-sectional study. BMC Womens Health. 2011 Dec 7;11:54. doi: 10.1186/1472-6874-11-54.

47. Sánchez Castro AC, Sáenz Orellana ADP. Conocimientos, actitudes y prácticas del autoexamen de mama en mujeres de 15 - 45 años que acuden a los consultorios de Planificación Familiar y Papanicolaou del Hospital Nacional Daniel Alcides Carrión, periodo agosto - diciembre del 2010. [tesis] Universidad Nacional Mayor de San Marcos, Facultad de Medicina. Lima, Perú; 2011.

48. Vela KV, Alarcón-Rozas AE. Estudio piloto sobre actitudes preventivas para cáncer de mama en mujeres de Sullana. Rev Med Hered. 2002;13(4):131-4.

49. Romaní F, Gutiérrez C, RamosCastillo J. Autoexamen de mama en mujeres peruanas: prevalencia y factores sociodemográficos asociados. Análisis de la Encuesta Demográfica de Salud Familiar (ENDES). An Fac Med. 2011;72(1):23-31.

50. Anderson BO, Yip CH, Smith RA, Shyyan R, Sener SF. Guideline implementation for breast healthcare in low-income and middle-income countries. Cancer. 2008 Oct 15;113(8 Suppl):2221-43. doi: 10.1002/ cncr.23844.

51. Claxton K. Centre for Health Economics. A Pilot Study of Value of Information Analysis to Support Research Recommendations for the National Institute for Health and Clinical Excellence. CHE Research Paper 4.York, UK: University of York 2005.

52. Knaul FM, Nigenda G, Lozano R, Arreola-Ornelas H, Langer A, Frenk J. Breast cancer in Mexico: an urgent priority. Salud Publica Mex. 2009;51 Suppl 2:s335-44.

53. Nigenda G, Caballero M, GonzálezRobledo LM. Barreras de acceso al diagnóstico temprano del cáncer de mama en el Distrito Federal y en Oaxaca. Salud Publica Mex. 2008;51(2):s254-62.

54. Goss P, Lee B, Badovinac-Crnjevic T, Strasser-Weippl K, ChavarriGuerra Y, St Louis J, et al. Planning cancer control in Latin America and the Caribbean. Lancet Oncol. 2013 Apr;14(5):391-436. doi: 10.1016/ S1470-2045(13)70048-2.

Correspondencia: Margarita Posso Dirección: : C/ St. Antoni M. Claret 167 Centro Cochrane Iberoamericano - Hospital de la Santa Creu i Sant Pau, Pabellón 18, CP 08025, Barcelona, España Teléfono: (+34) 935537814

Correo electrónico:mposso@cochrane.es 